\title{
NUEVAS EVIDENCIAS DE CAZADORES RECOLECTORES TERRESTRES DEL HOLOCENO MEDIO Y TARDÍO EN TIERRA DEL FUEGO: EL SITIO CABO MONMOUTH $20^{1}$
}

\author{
FLAVIA MORELLO', MANUEL ARROYO-KALIN", LUIS BORRERO ${ }^{* * *}$, JIMENA TORRES ${ }^{* * * *}$, \\ MAURICIO MASSONE ${ }^{* * * *}$, PEDRO CÁRDENAS" Y GABRIEL BAHAMONDE"
}

\section{RESUMEN}

Los hallazgos del sitio Cabo Monmouth 20 (CM20) representan nueva evidencia sobre ocupaciones del Holoceno medio (ca. 5.000 años AP) y tardío, en el norte de Tierra del Fuego. Se evalúa la información recopilada en excavaciones de sondeo, sintetizando los datos estratigráficos y contextuales, además de presentar un nuevo fechado realizado sobre material malacológico de origen antrópico, con una datación de $5.520 \pm 50$ años AP. Esta fecha confirma la antigüedad del sitio y la presencia de un depósito arqueológico inserto en un paleosuelo coetáneo.

PALABRAS CLAVE: Tierra del Fuego, Holoceno medio, cazadores terrestres.

\section{NEW EVIDENCE OF MIDDLE AND LATE HOLOCENE TERRESTRIAL HUNTER GATHERERS IN TIERRA DEL FUEGO: CABO MONMOUTH SITE}

\footnotetext{
ABSTRACT

The Cabo Monmouth 20 site represents new evidence of Middle and Late Holocene occupations (ca. 5.000 years BP) for the north of Isla Grande, Tierra del Fuego. In this paper we evaluate archaeological evidence from a test pit excavation, synthesize stratigraphic and contextual data, and present a new radiocarbon date $(5,520 \pm 50$ years $\mathrm{BP})$ on shell material of anthropic origin. The latter confirms the antiquity of the site, highlighting the presence of archaeological deposits in a co-eval palaeosol.

KEYWORDS: Tierra del Fuego, Middle Holocene, terrestrial hunters.

1 Estudio realizado en el marco del proyecto FONDECYT 1060020 "Arqueología de cazadores-recolectores de Tierra del Fuego: el proceso de colonización y el factor de aislamiento geográfico, durante el Holoceno temprano y medio".

* Centro de Estudios del Hombre Austral (CEHA), Instituto de la Patagonia, Universidad de Magallanes y Centro de Estudios del Cuaternario (CEQUA). flavia.morello@umag.cl. Av. Bulnes 01890, Punta Arenas, Chile.

* Centro de Estudios del Hombre Austral (CEHA), Instituto de la Patagonia, Universidad de Magallanes y McBurney Geoarchaeology Laboratory, Department of Archaeology, University of Cambridge. Inglaterra.

*** DIPA-IMIHICIHU, CONICET, Argentina.

Centro de Estudios del Cuaternario (CEQUA). Chile.

***** Museo de Historia Natural de Concepción, DIBAM. Chile.
} 


\section{INTRODUCCIÓN}

Las investigaciones iniciadas en el año 2006 en torno al proceso de colonización terrestre de Tierra del Fuego durante el Holoceno, son el marco de presentación de nueva evidencia recopilada sobre restos arqueológicos anteriores a la ocupación efectiva de la isla (ca 2.000 años AP, sensu Borrero 1989) en el sitio Cabo Monmouth 20.

Con el objetivo de discutir la dinámica cultural y las estrategias adaptativas de las poblaciones que habitaron la isla grande de Tierra del Fuego, Chile, se planteó la necesidad de generar nuevas estrategias metodológicas para mejorar el registro arqueológico conocido, entre otros aspectos. La metodología aplicada (cfr. Morello et. al. 2009) fue exitosa, llevándonos al estudio más detallado del sitio que se presenta a continuación.

El sitio Cabo Monmouth 20 (CM20) es un extenso yacimiento arqueológico descubierto en el marco de las prospecciones superficiales sistemáticas desarrolladas en la costa Norte y Oeste de Tierra del Fuego en el año 2006. Hasta el momento ha sido objeto de dos campañas de excavación de sondeo
(2007-2008), de una evaluación geoarqueológica (Arroyo-Kalin et. al. 2008) y de una tafonómica (Morello et. al. 2009).

El yacimiento está ubicado al sur de Porvenir (Fig. 1), sobre una barranca costera que en este sector tiene una altura estimada entre 6 y $10 \mathrm{msnm}$. Presenta una extensión aproximada de $1000 \mathrm{~m} \mathrm{~N}-\mathrm{S}$ y 150 m W-E (Massone et. al. 2007) y se emplaza en una terraza paleolacustre asociada al Avance $\mathrm{E}$ del Último Máximo Glacial (McCulloch 2007, 2008), que ha sido erosionada por el mar, conformando la barranca (Figs. 2 y 3).

La visibilidad del lugar es mala a nula, no obstante se observan artefactos dispersos en el perfil expuesto hacia la barranca costera y en superficie, en sectores alterados por roedores fosoriales (Ctenomys sp.) y por dos huellas vehiculares que cruzan la terraza. Se registraron diversos materiales líticos en superficie, como lascas, núcleos (incluyendo dos del tipo Levallois), raederas, fragmentos de bola, etc. También se observan algunos restos de fauna en superficie o en el perfil expuesto de la barranca, los que consideran guanaco (Lama guanicoe) -incluyendo un corte perimetral sobre epífisis datado-, lobo

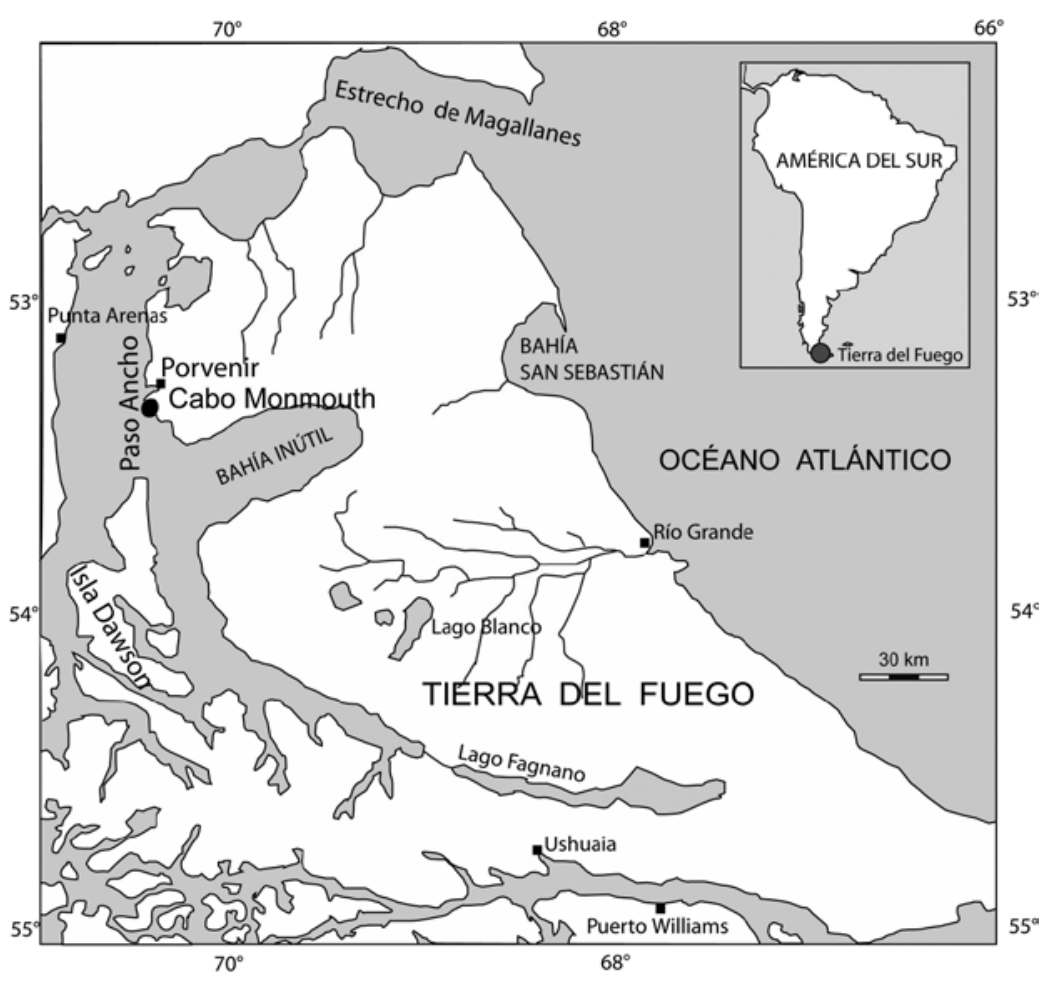

Fig. 1. Ubicación del sitio Cabo Monmouth 20. 


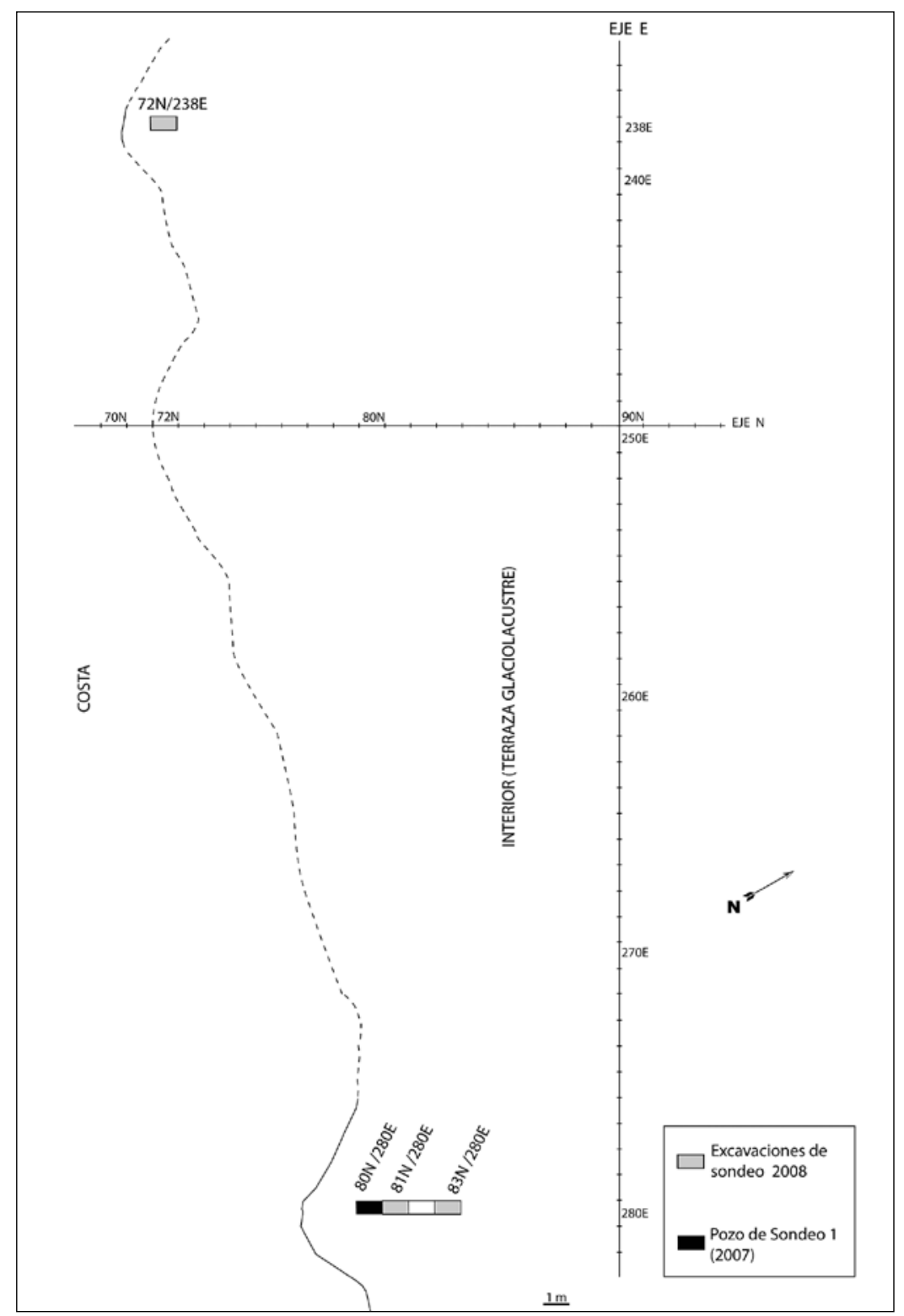

Fig. 2. Croquis de CM20 y la ubicación de las unidades excavadas.

marino, cetáceo y conchas dispersas y agrupadas en concentraciones.

Los fechados radiocarbónicos de dos muestras recolectadas en la campaña del año 2006 evidenciaron ocupaciones del Holoceno medio y tardío. Junto a las evidencias estratigráficas, estos datos sugerían que el sitio era de gran interés para una mejor comprensión del registro arqueológico de la Isla Grande. Estos primeros dos fechados se realizaron sobre muestras tomadas del perfil expuesto de la barranca costera, en el sector sur del sitio. Una datación tardía de $2.410 \pm$ 35 años AP (Ua-33947) fue realizada sobre un fragmento de corte perimetral sobre diáfisis y epífisis distal de radio-ulna derecha de guanaco, que provenía de la primera capa -superior- del perfil. De una segunda capa, posteriormente identificada como un paleosuelo que se expresa en varios puntos de la costa noroccidental de la isla, se obtuvo un fechado de $5.565 \pm 40$ años AP (Ua-33946), datando directamente el sedimento del paleosuelo (Morello et al. 2009). 


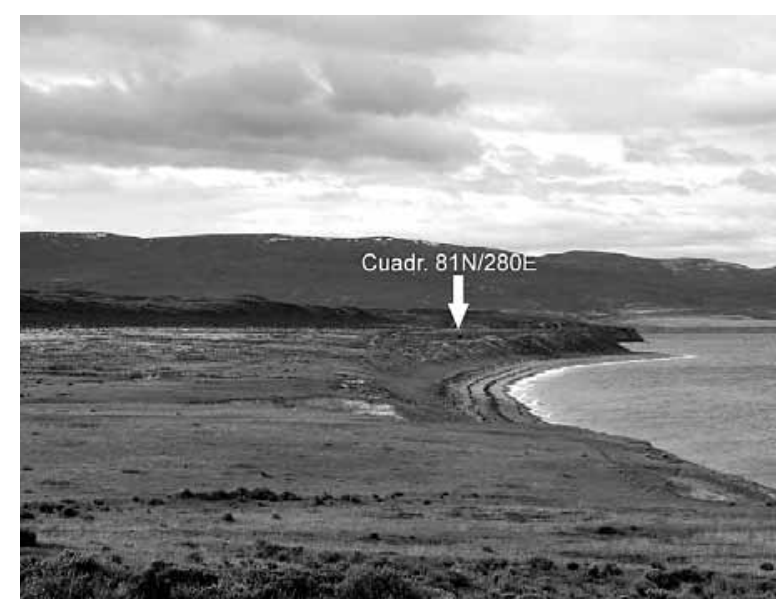

Fig. 3. Vista del sitio CM20, del lugar de sondeo (81N/280E) y de la terraza glaciolacustre donde se emplaza.

Con el objeto de verificar la información estratigráfica y contextual de estos dos fechados, se realizaron varios sondeos en dos sectores del sitio. Primero se sondeó en el año 2007 en la zona inmediatamente aledaña al lugar de recolección de las muestras datadas y, durante el año 2008, se excavó en otra zona cercana, en donde se observó una concentración de material cultural en un perfil erosionado de la barranca.

A continuación se presenta la metodología utilizada, los resultados obtenidos, una discusión de su relevancia y las conclusiones de estas excavaciones de sondeo.

\section{METODOLOGÍA}

La ubicación de las unidades de sondeo se situó en las cercanías de los sectores datados y al perfil expuesto del año 2006, al sur del yacimiento. La primera excavación realizada en el año 2007 se identificó como Pozo de Sondeo 1 (GPS 19F 0408061 S/ 4083509 E, Datum South American 69') pero luego fue asignado a una grilla de unidades de 1 $\mathrm{m}^{2}$ establecidas durante los trabajos del año 2008.

El yacimiento se subdividió en dos mitades arbitrariamente definidas por un eje imaginario orientado según el norte magnético y otro eje perpendicular a éste que se subdividió en Oeste y Este. Los tres ejes comienzan en un punto ubicado al azar y que corresponde a la medida 0 . Los Ejes se denominaron respectivamente Eje N, W y E. La zona de interés, estudiada en campañas anteriores, se ubica en la mitad Este (Fig. 2).
La subdivisión en ejes permitió generar un cuadriculado estándar para todo el yacimiento, dividiendo el área en unidades o cuadrículas de $1 \times 1 \mathrm{~m}$, denominadas según su distancia con respecto a los ejes diseñados. Así, por ejemplo, el Pozo de Sondeo 1 (año 2007) se denomina Cuadrícula 80N/280E. Esta excavación de sondeo abarca solamente la mitad oeste de la unidad, por lo que se decidió seguir excavando solo áreas de $1 \mathrm{~m}$ x $50 \mathrm{~cm}$, con su dimensión mayor orientada perpendicularmente a la barranca y en sectores muy cercanos al perfil expuesto. Así, durante el año 2008 se excavó la mitad oeste de las cuadrículas $81 \mathrm{~N} / 280 \mathrm{E}$ y $83 \mathrm{~N} / 280 \mathrm{E}$, conformando una trinchera semi-continua de $4 \mathrm{~m}$ de largo, que nos permitió conocer la distribución de materiales arqueológicos hacia el interior, alejándonos de la barranca.

Además de este sector, se realizó un sondeo preliminar en la cuadrícula $72 \mathrm{~N} / 238 \mathrm{E}$, a $42 \mathrm{~m}$ al oeste de la trinchera del eje 280E. Esta unidad también tuvo una extensión de $100 \times 50 \mathrm{~cm}$.

En todos los casos se excavó siguiendo niveles artificiales de $10 \mathrm{~cm}$ e intentando subdividir según capas naturales. Se harneó todo el sedimento con una malla de $4 \mathrm{~mm}$, recolectándose todo el material cultural, excepto los fragmentos de conchas y los fragmentos óseos de roedor, para los cuales se tomaron solamente algunas muestras para análisis radiocarbónicos y como registro. Una vez profundizado hasta un estrato estéril se dibujaron los perfiles de la excavación y se dispuso de una malla en la base y las paredes, y luego se tapó todo con el sedimento extraído.

Los materiales recolectados fueron sujeto de un análisis general, identificando las categorías de artefactos presentes, su frecuencia y peso, en el caso de los líticos, y la especie y unidad representada en el caso de los restos óseos.

\section{RESULTADOS}

Los resultados de las cuatro unidades excavadas se presentan a continuación.

\section{Cuadrícula 80N/280E (Pozo de Sondeo 1)}

La superficie del pozo presenta una cubierta vegetal total de pasto bajo y arbustos en los alrededores. En los primeros $10 \mathrm{~cm}$, luego de retirar la champa de pasto, se registra un sedimento café, suelto, con 
raicillas cuya matriz es de arena fina con limo y escasas gravas. Sólo se observan conchas aisladas en este nivel (choritos y mauchos -Mytilus sp. y Nacella sp., respectivamente) y escasos huesos de roedor.

Entre los $10-30 \mathrm{~cm}$ la matriz sedimentológica es la misma, correspondiendo al Estrato I. Comienzan a registrarse algunas lascas medianas a pequeñas y otros desechos, y siguen observándose conchas aisladas y dispersas, y escasos restos de roedor, a lo largo de la secuencia, pero entre los 20 y $40 \mathrm{~cm}$ la densidad de materiales líticos baja notablemente, lo que se observa en la Fig. 4, especialmente en las medidas de peso total.

Hacia los 30-40 cm de profundidad la matriz de sedimento cambia su color a un café más oscuro, orgánico, manteniendo su granulometría (arena fina y limo), correspondiendo a la transición hacia el Estrato II. Se observan escasos fragmentos de carbón y espículas dispersas. En cuanto al material arqueológico se registran algunas lascas, dos láminas pequeñas y aumenta la proporción de conchas dispersas y enteras (mauchos, Fisurella sp. y choritos). El material (conchas, carbón y líticos) se concentra en el nivel basal, entre aproximadamente 40-50 $\mathrm{cm}$ de profundidad, registrándose lascas de mayor tamaño y una raedera, bastantes conchas dispersas $y$ algunos escasos huesos de roedor.

Debemos destacar que en todos los niveles los materiales líticos se registraron en posición horizontal y no hay evidencia de procesos de disturbación, a

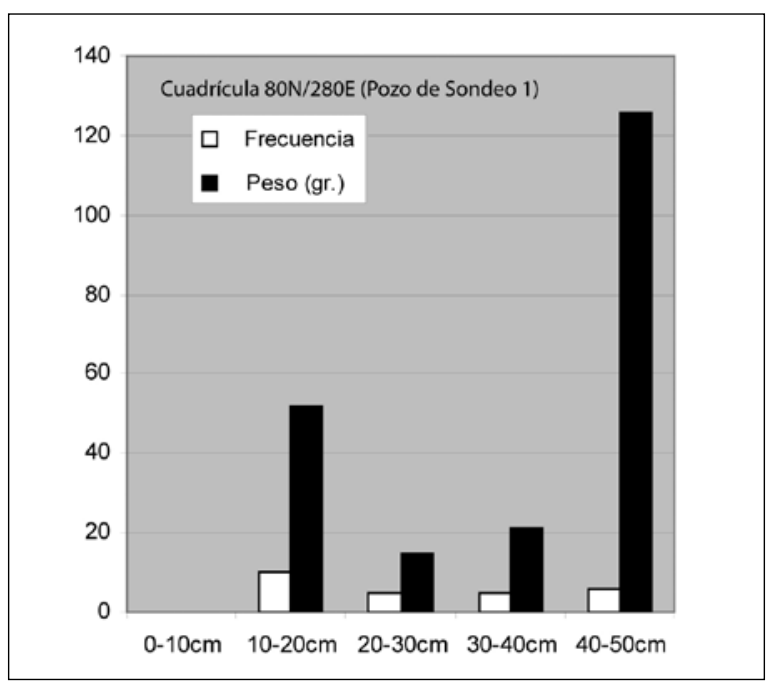

Fig. 4. Grafico de barras con frecuencia y peso total de materiales líticos de la cuadrícula $80 \mathrm{~N} / 280 \mathrm{E}$

(Pozo de Sondeo 1) por nivel artificial. excepción de los escasos restos de roedores, dominados por coruro.

Hacia los $47 \mathrm{~cm}$ de profundidad, en la esquina SW de la unidad, se registra un sedimento de color claro y de origen glaciar. Se sigue excavando la capa de arena fina con limo de color café oscuro, aunque hacia la base cambia y se presenta más compacta y con más limo. La excavación continua hasta despejar el estrato de sedimento glaciar en todo el pozo. El nuevo Estrato III esta compuesto de un limo arcilloso con concentraciones pequeñas y discontinuas de tefra que corresponderían a el volcán Reclus (com pers. R. McCulloch 2006). Entre los 50-60 cm de profundidad se reduce la excavación de la unidad a la mitad norte $(50 \times 50 \mathrm{~cm})$, llegando a despejar otra capa de finales del Pleistoceno. En este sector el pozo llega a una profundidad máxima de $76 \mathrm{~cm}$.

\section{Cuadrícula $81 \mathrm{~N} / 280 \mathrm{E}$}

La cubierta vegetal en esta zona es total (pasto bajo y arbustos en los alrededores) y el estrato bajo la champa de pasto corresponde a una arena fina con limo y escasas gravas, color café claro.

Entre los primeros niveles artificiales, 0-35 $\mathrm{cm}$, se registran restos culturales dispersos, principalmente material lítico (lascas, microdesechos y fragmentos), algunas conchas de chorito y maucho y escasos huesos de roedor. El material se concentra principalmente en el nivel 10-20 cm (Fig. 5).

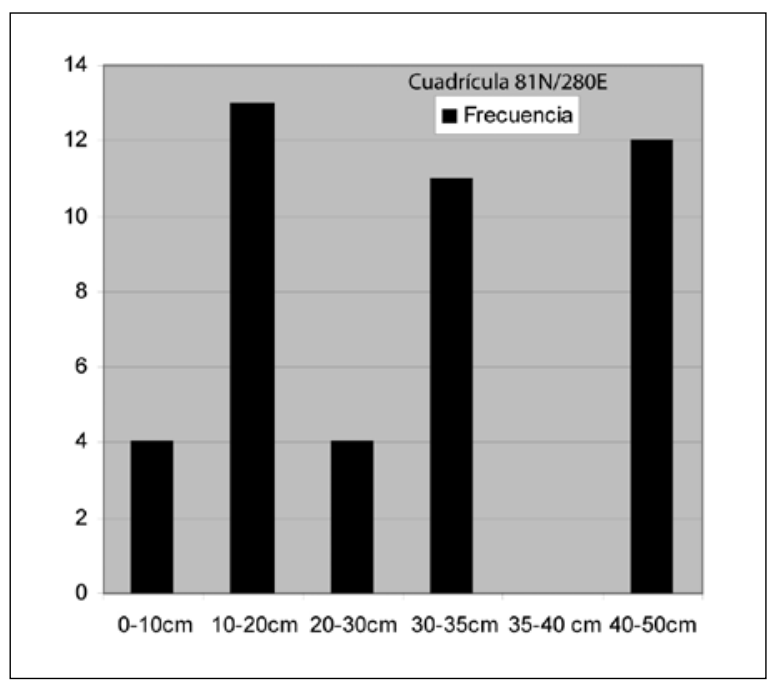

Fig. 5. Frecuencia de artefactos líticos recuperados en la cuadrícula $81 \mathrm{~N} / 280 \mathrm{E}$ por nivel artificial. 


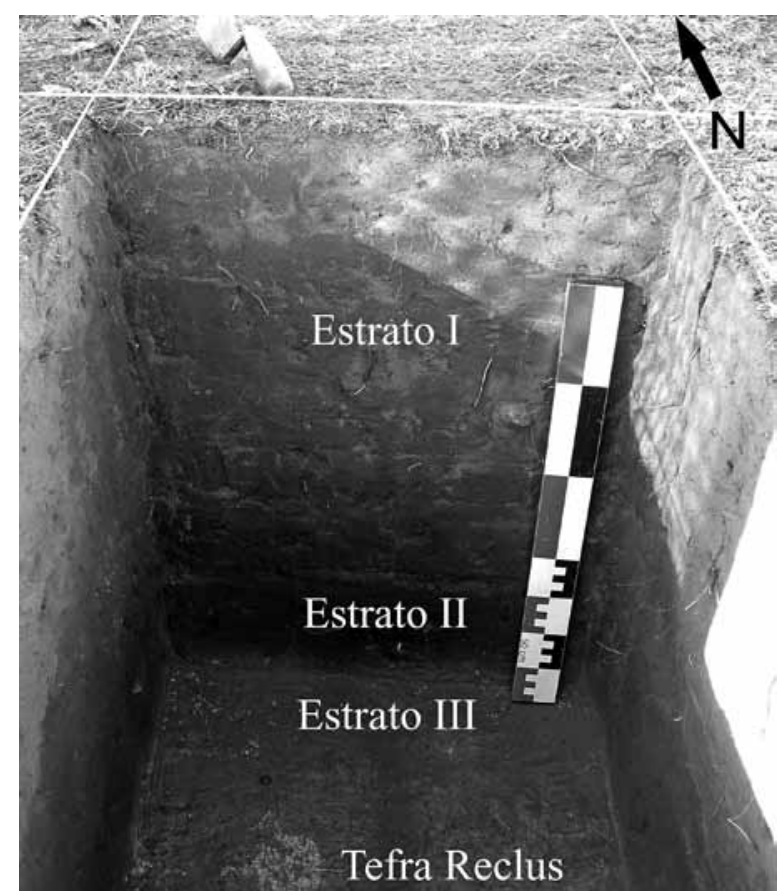

Fig. 6. Perfil Norte de la Cuadrícula 81N/280E.

Hacia los $30 \mathrm{~cm}$ se observa una disminución en la cantidad de materiales, con una alza entre los 30-35 cm de profundidad y un nivel estéril de restos líticos entre los $35-40 \mathrm{~cm}$ (solo se observaron conchas aisladas).

Luego, entre los $40-50 \mathrm{~cm}$ se registra nuevamente material lítico, con una concentración mayor que la de los niveles anteriores, observándose lascas internas, secundarias y fragmentos, un guijarro con fractura bipolar y un percutor. El sedimento asociado presenta un leve aumento en la cantidad de gravas, es más compacto y de un color más oscuro, con raicillas dispersas. Corresponde a la transición hacia el Estrato II (paleosuelo) - Fig 6.

El nivel $50-60 \mathrm{~cm}$ se relaciona con el Estrato II, un paleosuelo enterrado, compuesto de una arena fina y más compacta, con abundante material orgánico y de color oscuro. Hacia la base se presenta el Estrato III, de origen glaciar, también estéril, y que registra concreciones pequeñas de color blanco que corresponden a la tefra del Reclus.

\section{Cuadrícula $83 N / 280 E$}

La cubierta vegetal en esta unidad también es total y se presenta una estratigrafía muy similar a

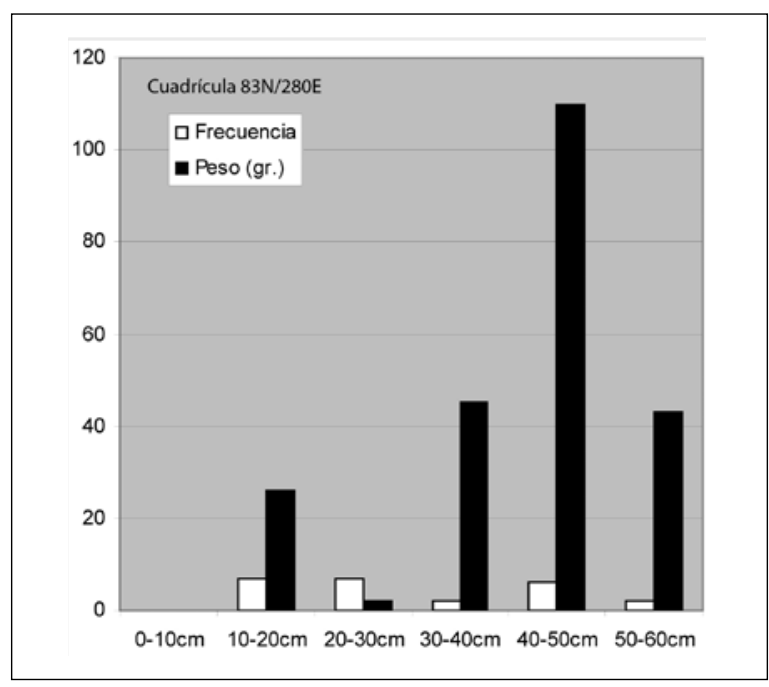

Fig. 7. Frecuencia y peso de restos líticos de la cuadrícula $83 \mathrm{~N} / 280 \mathrm{E}$ por nivel artificial.

las anteriores. En asociación con el Estrato I, entre los 0-40 cm, se observan algunos materiales líticos (lascas internas, secundaria y otros desechos), escasos restos de roedor y conchas aisladas. Aunque en los primeros $10 \mathrm{~cm}$ no se registra ningún artefacto lítico.

Hacia los $40 \mathrm{~cm}$ de profundidad se recolecta un núcleo y se observa un alza en la densidad de materiales líticos, registrándose en los niveles 40-55 cm artefactos más grandes como una lasca secundaria, lascas internas, un desecho de bola de gabro y desechos de talla indeterminados (Fig. 7). Se recolecta una astilla ósea de Mammalia en muy mal estado de conservación.

Entre los 55-60 $\mathrm{cm}$ se pasa casi de manera imperceptible al Estrato II, ya que el paleosuelo no presenta un espesor mayor a $2 \mathrm{~cm}$ y por la humedad de los sedimentos se hace difícil identificarlo al excavar. No se registran materiales culturales asociados a estos últimos $5 \mathrm{~cm}$. El Estrato III, de origen glacial, se presenta bajo los $60 \mathrm{~cm}$ de profundidad, siendo también estéril.

Al terminar esta última unidad del eje $280 \mathrm{~N}$, el conjunto de estas tres unidades excavadas conforman una transecta perpendicular a la barranca que permite tener una visión sobre la distribución estratigráfica y de restos culturales desde el perfil erosionado hacia el interior o norte de la terraza glaciolacustre, donde se extiende el sitio CM20. Entre los resultados comunes de estas tres cuadrículas destaca una reiteración en el hallazgo de dos peaks 


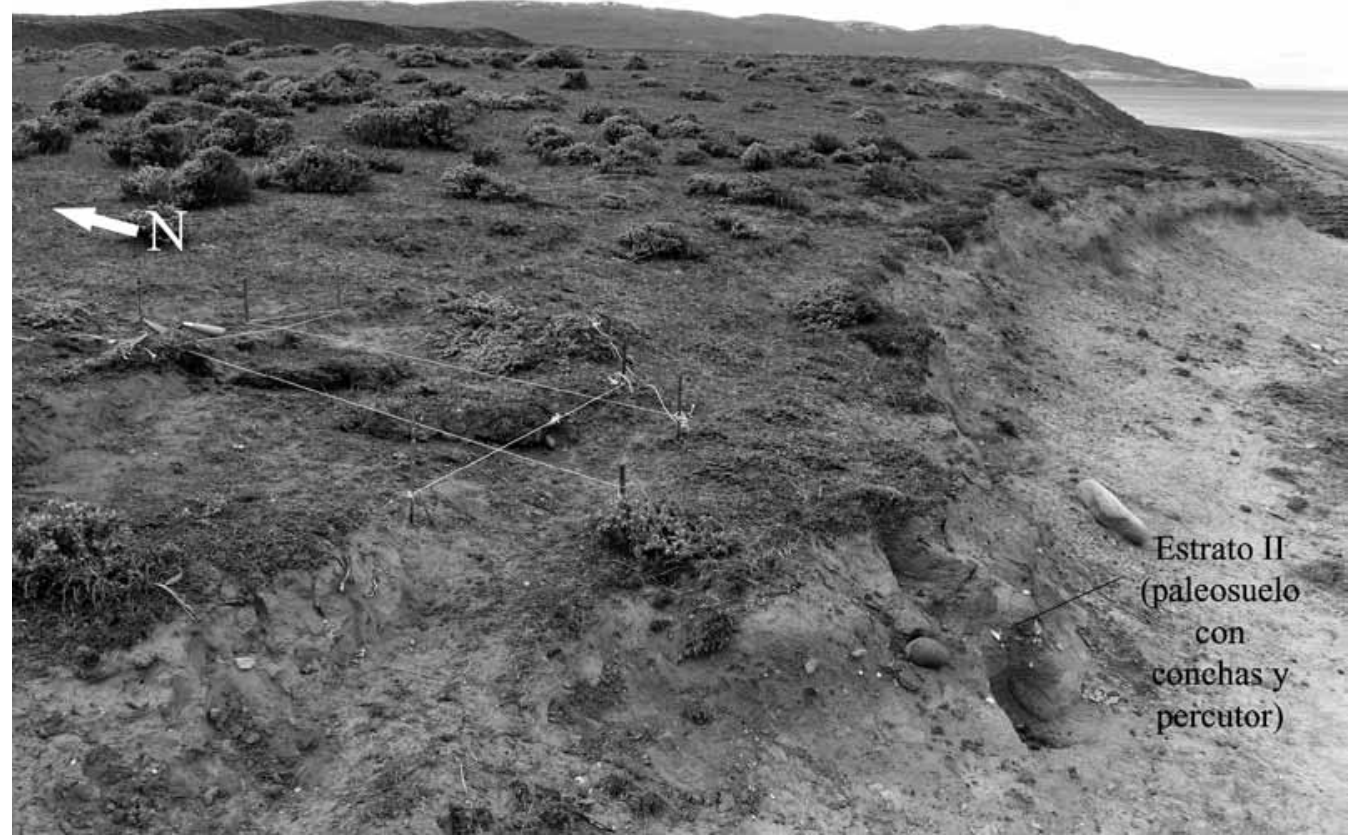

Fig. 8. Vista de la cuadrícula $72 \mathrm{~N} / 238 \mathrm{E}$ al inicio de la excavación y el perfil expuesto por la barranca en el que se observa la extensión continua del paleosuelo y su asociación de restos líticos y conchas.

de concentración de restos líticos, uno aproximadamente entre los $10-20 \mathrm{~cm}$, que coincide bien con el estrato y el depósito arqueológico fechado en 2.410 años AP y, un segundo, con mayor concentración de artefactos y de mayor tamaño, los que se apoyan e insertan en el interior del paleosuelo - Estrato II - y que se relaciona directamente con la datación de éste con ca. 5.500 años AP.

La observación de la estratigrafía general de esta transecta indica que la capa de paleosuelo se adelgaza al alejarse del perfil de la barranca, hacia el Norte y el examen del paleosuelo en diversas exposiciones revela una superficie enterrada que no es paralela a la actual, también sugiriendo una diferente paleotopografía que se inclina hacia la barranca.

\section{Cuadrícula $72 N / 238 E$}

La vegetación cubre alrededor del 50\% de la superficie de esta unidad, generando notorias diferencias de altura, con cavidades de erosión en distintos sectores de la cuadrícula. Es por esto que antes de comenzar la excavación se saca la champa de pasto y se nivela horizontalmente en relación a los sectores erosionados y luego se comienza a excavar con niveles artificiales (Fig. 8). En esta extracción de la champa se registra en harnero un fragmento de lasca y microdesechos.

Entre $0-20 \mathrm{~cm}$ se registra el Estrato I, con las mismas características que en las cuadrículas anteriores. Los restos recuperados corresponden a lascas internas, microdesechos, una lasca secundaria grande, un núcleo, un cuchillo y otros desechos indeterminados, también se registran escasas conchas (chorito y maucho), huesos de roedor dispersos y una patela de guanaco con abundantes marcas de radículas. Hacia la esquina $\mathrm{NE}$, en el nivel 0-10 cm, se identifica una fosa de roedor. En la base del nivel 10-20 cm, pero solo en la mitad Sur de la unidad, comienza a vislumbrarse la transición del Estrato II (paleosuelo).

Asociada a esta capa de transición, arena fina con mayor porcentaje de limo y algunas gravas, se registra un interesante conjunto de materiales culturales. Presenta una mayor frecuencia de conchas de choritos y mauchos, y se observan una lasca secundaria grande, un núcleo y una lasca de dorso natural grande apoyando sobre esta capa más oscura. 


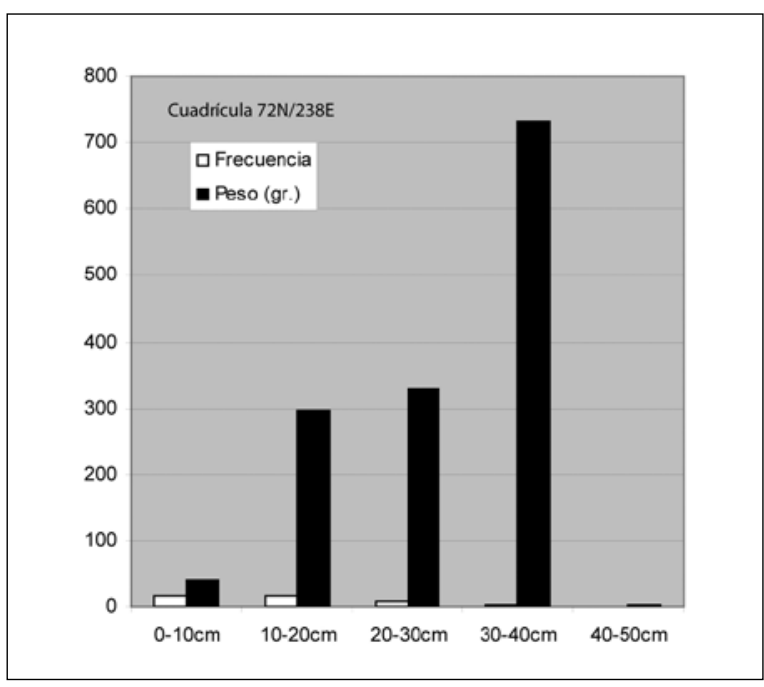

Fig. 9. Grafico de barras con frecuencia y peso total de materiales líticos de la cuadrícula $72 \mathrm{~N} / 238 \mathrm{E}$ por nivel artificial.

También lascas internas, microdesechos y algunas espículas de carbón dispersas, todo en la mitad sur de la cuadrícula.

En la mitad norte, en el nivel $20-30 \mathrm{~cm}$, también comienza a observarse esta capa de tran- sición de arena limosa café oscuro y se registra otra concentración de grandes líticos (lasca primaria, lasca de dorso natural y fragmento) y en el sector sur de la unidad siguen apareciendo líticos como un guijarro astillado y una lasca pequeña. En este nivel la existencia de una concentración de conchas es evidente, aunque siguen presentándose de manera dispersa y registrándose espículas de carbón y una de colorante ocre. Hacia la base del nivel, en la zona norte, se recolecta una raedera y un desecho de cuarzo, entre otras piezas (Fig. 9).

En general, todos los materiales se presentan en posición horizontal o ligeramente oblicua, lo que se podría asociar a la pendiente de la terraza, también observada en la distribución horizontal de las capas naturales, que se inclinan o descienden levemente hacia la barranca costera (Sur) - Fig. 10. Esta observación es extrapolable a todas las unidades excavadas en CM20.

La excavación del nivel artificial 30-40 cm registra la aparición del Estrato II, paleosuelo de arena limosa muy oscura y compacta que se asocia a concentraciones de conchas, mezcladas con lentes carbonosos y de cenizas, y conchas quemadas, además de materiales culturales y escasas espículas

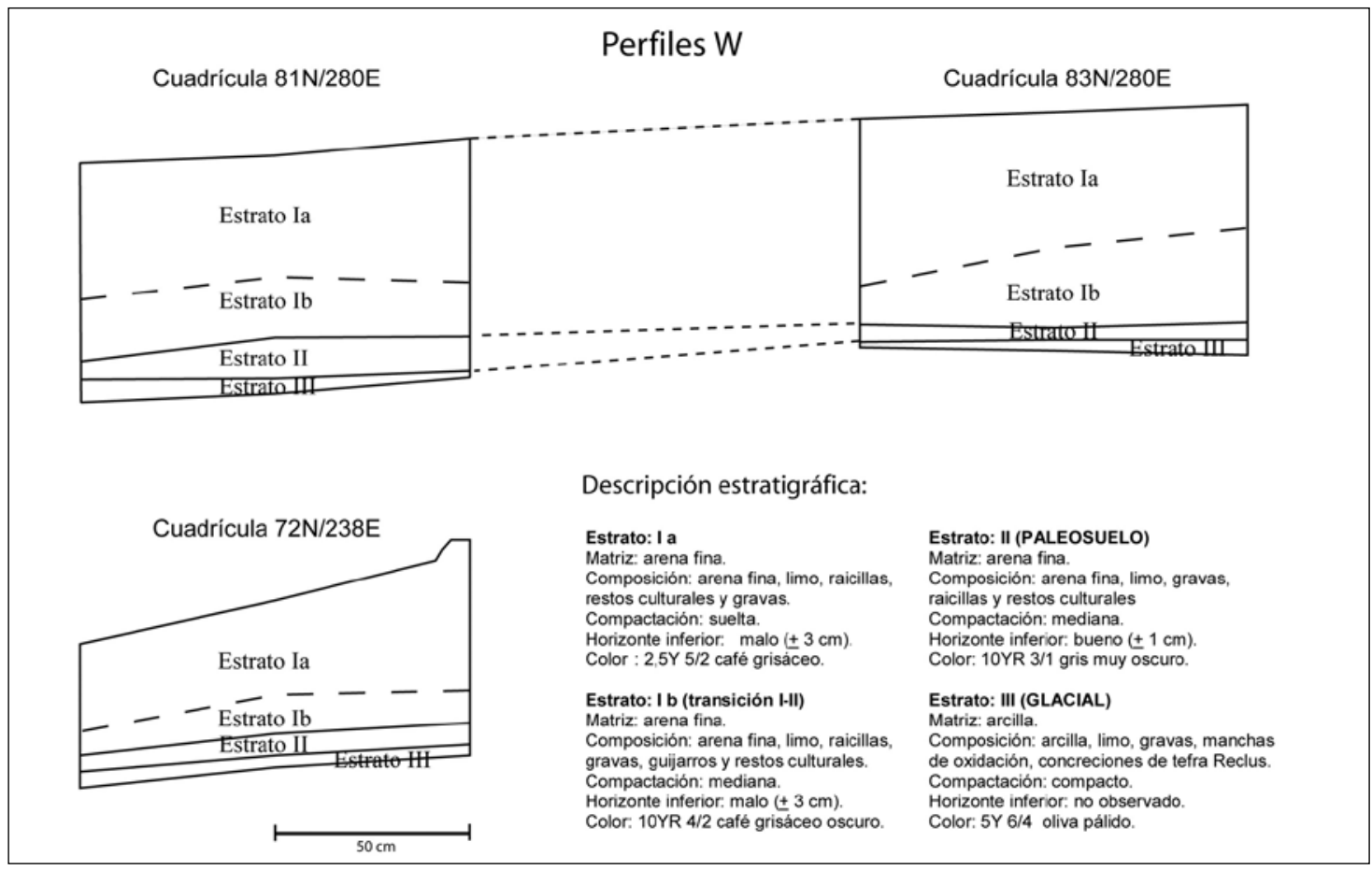

Fig. 10. Perfiles estratigráficos de las unidades excavadas en el sitio. 
de carbón (aunque el sedimento es de contenido altamente orgánico). Entre los restos líticos destaca un núcleo Levallois simplificado (sensu Pigeot 2003), una lasca secundaria y lascas internas. Una muestra de conchas (Mytilus sp.) tomadas de este rasgo, de la zona sur de la unidad, fue datada en $5.520 \pm 50$ años AP (Beta-255156), lo que ubica el contexto arqueológico de manera contemporánea con el paleosuelo previamente datado (cfr. Supra).

La excavación de este nivel registra algunas astillas óseas en el sector norte del sondeo y luego una concentración de materiales que están insertos en la parte superior del paleosuelo. Se trata de un hueso de cetáceo que podría presentar huellas de corte pero la gran cantidad de marcas de radículas en su superficie no nos permite confirmar esta observación. Otro fragmento óseo correspondiente a una diáfisis de hueso largo de guanaco se presenta en las mismas condiciones de alteración y asociado a dos lascas.

En el mismo nivel, bajo el paleosuelo, comienza a vislumbrarse el Estrato III, de origen glaciar. En la parte superior del nivel 40-50 cm, en la mitad sur de la unidad, se descubre esta capa glaciar. Más abajo, se extiende en toda la cuadrícula, una vez terminada la excavación del paleosuelo. En este punto se registra una lasca interna. En el Estrato III se detecta solamente un fragmento de diáfisis de hueso largo de guanaco con coloración negra (quemada o manganeso), apoyado en su parte superior.
Al excavar algunos centímetros del Estrato III, arcilla de color claro, se distinguen manchas como concreciones de color blancuzco que corresponden a concentraciones de tefra, tratándose de la erupción del volcán Reclus. La ceniza volcánica se observa a lo largo de la barranca en el área estudiada. No se registra material arqueológico asociado y se concluye la excavación.

\section{Materiales Óseos y Líticos}

La densidad de materiales arqueológicos en las unidades excavadas es mediana, llegando a un promedio de 74 restos por metro cuadrado, incluyendo todos los niveles artificiales. Si consideramos sólo los restos de fauna, la densidad puede considerarse bajísima, más si se excluyen los escasos restos de coruro y las conchas dispersas presentes en todas las unidades. En las cuadrículas 80N/280E y $81 \mathrm{~N} / 280 \mathrm{E}$ no se registró ningún resto óseo, luego en la unidad 83N/280E sólo se recolectó una astilla de Mammalia. Con relación, destaca la diversidad de restos de fauna encontrados en la unidad 72N/238E, pues allí se recuperar un resto de cetáceo, tres huesos de guanaco y algunas astillas de Mammalia, pero sigue siendo llamativa la escasa presencia de restos óseos.

Por consiguiente, la mayoría de los materiales recuperados son restos líticos, principalmente desechos de talla, aunque también se registraron algunos instrumentos formatizados por retoque marginal (Tabla 1). Los derivados de núcleo presentes en las distintas

Tabla 1. Frecuencia de materiales líticos según categoría tecno-morfológica y cuadrícula.

\begin{tabular}{|c|c|c|c|c|c|}
\hline \multirow{2}{*}{ CATEGORÍA } & \multicolumn{4}{|c|}{ CUADRÍCULA } & \multirow[b]{2}{*}{ TOTAL } \\
\hline & $80 \mathrm{~N} / 280 \mathrm{E}$ & $81 \mathrm{~N} / 280 \mathrm{E}$ & $83 \mathrm{~N} / 280 \mathrm{E}$ & $72 \mathrm{~N} / 238 \mathrm{E}$ & \\
\hline raedera & & & & 1 & 1 \\
\hline cuchillo & & & & 1 & 1 \\
\hline percutor & & 1 & & & 1 \\
\hline núcleo & & & 1 & 3 & 4 \\
\hline lasca primaria & & 4 & & & 4 \\
\hline lasca secundaria & 4 & 2 & 4 & 6 & 16 \\
\hline lasca dorso natural & 1 & & & 2 & 3 \\
\hline lasca interna & 14 & 20 & 6 & 13 & 53 \\
\hline lámina & 2 & & & & 2 \\
\hline lasca de retoque & 1 & & & & 1 \\
\hline lasca bifacial & & & & 1 & 1 \\
\hline microdesecho & & 10 & 1 & 9 & 20 \\
\hline desecho de bola & & & 1 & & 1 \\
\hline desecho indeterminado & 4 & 6 & 11 & 12 & 33 \\
\hline TOTAL & 26 & 43 & 24 & 48 & 141 \\
\hline
\end{tabular}


cuadrículas señalan el desarrollo de actividades de talla, estando representadas las primeras etapas de desbaste de núcleos, pero destacamos el escaso registro de la etapa final de retoque de instrumentos sobre lasca y la casi ausencia de trabajo bifacial.

Las actividades de desbaste incluyen la presencia de un núcleo Levallois simplificado (sensu Pigeot 2003), asociado a la fecha del paleosuelo de ca. 5.000 años AP, y similar a las dataciones del mismo tipo de método de desbaste registrado en Ponsonby (Legoupil 2003). Al respecto, destacamos que en superficie se registraron al menos dos casos de núcleos Levallois recurrente.

\section{Geoarqueología}

El análisis de cortes expuestos en la barranca costera sugiere que la estratigrafía relevada en la excavación no constituye una excepción a escala mayor del paisaje. El llamado Estrato II corresponde a un paleosuelo detectable a todo lo largo de la explanada que constituye el sitio Cabo Monmouth. El estudio micromorfológico del mismo muestra un suelo de microestructura granular, textura limo-arcillosa, y porosidad y agregados con claras indicaciones de bioturbación. Depositado en el mismo se observan raicillas reemplazadas por sesquióxidos y también fragmentos microscópicos de concha y carbón. La presencia de estos últimos apoya la interpretación de que los artefactos detectados en la excavación arqueológica son contemporáneos con la formación del paleosuelo. Inmediatamente bajo este último, en el sedimento arcillo-limoso que constituye el Estrato III, se observa un fino lente de sedimento más claro. Las observaciones microscópicas confirman características de una ceniza volcánica de color amarillento. Estudios en ejecución nos permitirán confirmar si ésta resulta comparable con la tefra del volcán Hudson, lo que resulta plenamente compatible desde un punto de vista cronológico. El Estrato III aparece como un sedimento limo-arcilloso de coloración tipo gley que en el microscopio no presenta estratificación y sí, en cambio, porosidades que sugieren actividad biológica. Hacia la parte inferior del perfil hay un cambio hacia una estructura limoarenosa fina que presenta evidencias de la presencia de la tefra del Reclus. Ello sugiere una transición desde un régimen de sedimentación caracterizado por depositación eólica a uno en el que el agente principal es el agua, seguido de la implantación de condiciones anaeróbicas. Estas observaciones apoyan parcialmente la presencia de un lago glaciar antes de la depositación de la tefra del Reclus, ca. 12.500 años AP (McCulloch 2007, 2008).

\section{Tafonomía}

Resulta importante incorporar una evaluación tafonómica, pero esta necesidad se vuelve imperativa cuando se está presentando un caso novedoso. Como se ha dicho, en Cabo Monmouth, una pradera con arbustos, la visibilidad es muy baja, al punto que solo en ca. 9\% de los casos analizados en una prospección sistemática de la localidad, basada en distintas tácticas, pudo ser juzgada como alta (Morello et. al. 2007). Por otra parte la visibilidad disminuye al alejarse del borde de la barranca hacia el interior, como lo evidencian los pozos de transectas C-5-C-6, D-6 y E-2 a E-6 (Morello et. al. 2007) de la prospección realizada en el año 2007.

Las observaciones tafonómicas indican que el suelo es poco penetrable y son bajas las probabilidades de mezcla de huesos arqueológicos con materiales recientes. En otras palabras, aunque existan condiciones de buena depositación de restos, eso no parece crear un riesgo excepcional de mezcla. Las mezclas sólo parecen ocurrir en sectores erosionados. Si bien estos son cada vez más amplios, se puede sostener que su acción de mezcla es controlable eligiendo -como se hizo- sectores no erosionados para excavar.

En el caso de Cabo Monmouth 20, como en muchos otros a lo largo de este sector de costa, las verdaderas asociaciones secundarias se generan al pie de la barranca y son el resultado del retroceso de la misma. Los casos de contaminación más esperables, en realidad, ocurren alejándose de la costa, en los sectores de actividad de Ctenomys sp., zona en la que se detectó la existencia de una corurera activa, aunque dominada -como es usual- por madrigueras abandonadas.

Todos los sondeos presentaron restos de roedores en distintos niveles, especialmente entre 0-20 cm, y también en niveles inferiores asociados al paleosuelo. Son casos con asociaciones líticas y óseas arqueológicas verticalmente discretas, y sin evidencias de disturbación "a excepción de los escasos restos de roedores" (Morello y Cárdenas 
2008: 60). En otras palabras, (1) la escasa acción de los roedores no afectó al paleosuelo, (2) la presencia de los restos de roedor es cotérmino con la de los elementos culturales, pero siempre indicando actividad animal muy poco intensa.

La ausencia de indicadores culturales sobre restos de roedores permite considerarlos un artefacto de depositaciones penecontemporáneas o posteriores a la instalación humana. En pocas palabras, los roedores aparecen como agentes poco importantes en este depósito. La posición horizontal de los hallazgos, junto con la ausencia de marcas de roedor sobre los huesos -el principal agente "mezclador" potencial-, robustecen la credibilidad del conjunto.

A pesar de esta situación de poca contaminación, el sitio nos presenta un caso en el que la complejidad de la historia tafonómica, limita no solo la inferencia de conducta y, por extensión dietética -qué recursos llevan huellas de corte- (Blumenschine y Pobiner 2007), sino directamente la cultural. Un ejemplo es el caso del hueso de cetáceo. Sin embargo, aunque este no ofrece evidencia creíble de significado cultural -sea para consumo o como materia prima-, su contexto si lo indica. Hemos visto que, a diferencia de otros casos en la costa del estrecho de Magallanes, es muy difícil explicar su aparición en el sitio sobre un sustrato no marino excepto a través de mediación cultural.

En resumen, las posibilidades de que la mezcla reciente haya afectado mucho a los conjuntos de Cabo Monmouth 20 son bajas. Los materiales ubicados han sido dispersos, pero constituyen un innegable testimonio de ocupación hacia el Holoceno medio.

\section{CONCLUSIONES}

Los estudios iniciales efectuados durante el año 2007, en especial el análisis geoarqueológico y el Pozo de Sondeo 1 (cuadrícula 80N/280E), resultaron positivos en cuanto a confirmar la presencia de un depósito arqueológico con artefactos líticos y conchas asociadas a un fechado cercano a 5.500 años AP. El contexto esta incorporado estratigráficamente en el interior de un paleosuelo que tiene la datación mencionada. Además se corroboró la estrecha concordancia entre la estratigrafía registrada en los perfiles expuestos en la barranca costera - previamente datados en el año 2006 - y las excavaciones de sondeo.
Considerando la coherencia estratigráfica en conjunto con la existencia de dos concentraciones de materiales líticos, uno en el nivel $10-20 \mathrm{~cm}$ y otro en la base entre los 40 y $50 \mathrm{~cm}$ de profundidad, es posible concluir que en el sector registramos dos ocupaciones estratigráficamente superpuestas y asociadas a los fechados de 2.410 y 5.565 años AP, respectivamente. Sin embargo, la densidad de materiales óseos es baja y hay escasa presencia de restos óseos de claro origen antrópico. No así, los artefactos líticos están bien representados e incluyen diversas actividades de talla.

La información recopilada en la excavación del sondeo 72N/238E aporta, además, evidencia clara de la existencia de un agregado de concentraciones discontinuas en el espacio y correspondiente al registro arqueológico del Holoceno medio. Esto, considerando un efecto reservorio aproximado de 400 a 500 años para esta zona del estrecho de Magallanes da una data de ca. 5.000 años AP para el contexto arqueológico (cfr. San Román et. al. 2009). Según lo muestran los sondeos realizados en dos sectores del yacimiento, el depósito se encuentra asociado a un paleosuelo (5.565 años AP) que presenta una extensa distribución en la localidad, y a ocupaciones posteriores que pueden preliminarmente ubicarse alrededor de los 2.410 años AP.

Las excavaciones realizadas en las cuadriculas $81 \mathrm{~N} / 280 \mathrm{E}$ y $83 \mathrm{~N} / 280 \mathrm{E}$ permiten aclarar, en este sector, que una concentración de materiales líticos se presenta en la capa de transición directamente sobre el paleosuelo, pero el interior de este se registró estéril, probablemente debido a su poco espesor. Además, es interesante destacar que la capa de paleosuelo se adelgaza a medida que los sondeos excavados se alejan del perfil de la barranca, hacia el N (Fig. 10). La conservación de restos óseos, en general, es muy mala y podríamos suponer que este adelgazamiento del paleosuelo podría estar asociado a procesos de erosión y tal vez explicar la casi total ausencia de huesos en estas unidades.

Para Cabo Monmouth 20 el registro predominante de materiales en posición horizontal, extensiva a todas las unidades, sugiere que ha habido poca movilidad vertical en el caso del componente del Holoceno medio del sitio. Pero la presencia de al menos una fosa de roedor clara en el Estrato I de la 
excavación de la cuadrícula 72N/238E, además de muchas otras observadas dispersas en la superficie del yacimiento y la presencia constante de huesos de roedor dispersos en los sondeos, aunque en pequeñas cantidades, sugiere que pueden haber procesos de disturbación no detectados, especialmente asociados a los niveles entre $0-30 \mathrm{~cm}$ de profundidad. La notable presencia de marcas de radículas en los huesos de guanaco y cetáceo hallados en la transición del horizonte superior y en el interior del Estrato II - paleosuelo - de esta última unidad, es un indicio de la importancia de este factor en los procesos de disturbación del contexto datado en ca. 5.000 años AP y, probablemente, en la explicación de la mala preservación y baja cantidad de restos orgánicos conservados en este depósito. No obstante, la evaluación tafonómica del sitio y la localidad, en general, indican que las posibilidades de mezcla con materiales recientes es baja.

Las dataciones del paleosuelo registrado en Cabo Monmouth corresponden con condiciones más húmedas, ya que para el Holoceno mediotardío el incremento en la precipitación condujo al desarrollo de un bosque de Nothofagus más cerrado, como el que se observa actualmente en las cercanías de Cordillera Darwin (cfr. McCulloch y Morello 2009). Tanto la presencia del paleosuelo como la abundancia de marcas de raíces en los restos óseos, y su mala conservación, apoyan la presencia de diferencias en la vegetación actual con respecto a la que debió predominar en el Holoceno medio en la localidad.

El interés de los contextos descritos, su antigüedad cercana a 5.000 años y su cercanía a una barranca costera en constante y rápida erosión, hacen que la excavación ampliada de una o dos de estas concentraciones de ocupaciones del Holoceno medio sean una prioridad para las labores de terreno de los próximos años.

\section{AGRADECIMIENTOS}

Junto con agradecer el financiamiento de FONDECYT 1060020, quisiéramos dar nuestra gratitud a las personas que colaboraron en las campañas de terreno: Magallanes Rovira y Manuel Román. Además, los oportunos comentarios de Fabiana Martin.

\section{BIBLIOGRAFÍA}

\section{ARROYO-KALIN, M, F. MORELLO, C. FRENCH Y P. CÁRDE-}

NAS. 2008. Sedimentos y paleosuelos: una aproximación geoarqueológica a la evolución del paisaje fueguino durante el Holoceno. En: Informe de Avance, Año 2, Proyecto FONDECYT 1060020, pp. 78-86. MS

BORRERO, L. 1989. Spatial Heterogeneity in Fuego-Patagonia. En: Archaeological Approaches to Cultural Identity, Shenan editor. One World Archaeology.

BLUMENSCHINE, R. y B. POBINER, 2007. Zooarchaeology and the Ecology of Oldowan Hominin Carnivory. En: Evolution of the Human Diet. Editado por P.S. Ungar, pp. 167-190, Oxford University Press, Oxford.

LEGOUPIL, D. 2003. Cazadores-Recolectores de Ponsonby (Patagonia austral) y su paleoambiente desde VI al III milenio A.C. editado por D. Legoupil. Universidad de Magallanes MAGALLANIA Vol. 31, número especial, Punta Arenas.

MASSONE, M., F. MORELLO, E. CALÁS, C. HUIDOBRO, G. BAHAMONDE, Y I. MARTINEZ. 2007. Prospección arqueológica superficial en la costa norte y noroccidental de Tierra del Fuego. En: Informe de Avance, Año 1, Proyecto FONDECYT 1060020, pp. 53-65. MS.

MCCULLOCH, R. D. 2007. Field Report 2006. En Informe de Avance, Año 1, Proyecto FONDECYT 1060020 , pp. 13-22. MS.

-2008. Field Report 2007. En: Informe de Avance, Año 2, Proyecto FONDECYT 1060020. MS.

MCCULLOCH, R. Y F. MORELLO. 2009. Evidencia glacial y paleoecológica de ambientes tardiglaciales y del Holoceno temprano. Implicaciones para el poblamiento temprano de Tierra del Fuego. En: Arqueología de Patagonia: una mirada desde el último confín, editado por $\mathrm{M}$. Salemme, F. Santiago, M. Álvarez, E. Piana, M. Vázquez y M.E. Mansur. Editorial Utopías, Ushuaia. En prensa. MORELLO, F. y P. CÁRDENAS. 2008. Excavación de sondeo en Cabo Monmouth 20, Tierra del Fuego. En: Informe de Avance, Proyecto FONDECYT 1060020, $2^{\circ}$ año, pp. 57-63. MS.

MORELLO, F., M. ARROYO-KALIN, M. MASSONE, I. MARTINEZ, C. HUIDOBRO, G. BAHAMONDE y P. CÁRDENAS. 2007. Evaluación de técnicas sub-superficiales de prospección: prospección de múltiples etapas de las costas de la transgresión marina del Holoceno medio en el Norte y Oeste de Tierra del Fuego. En: Informe de Avance, Proyecto FONDECYT 1060020, 1er año, pp. 29-52. MS. 
MORELLO, F.; L. BORRERO, J. TORRES, M. MASSONE, M. ARROYO- KALIN, R. MCCULLOCH, E. CALAS, M. LUCERO, I. MARTÍNEZ Y G. BAHAMONDE. 2009. Evaluando el registro arqueológico de Tierra del Fuego durante el Holoceno temprano y medio. En: Arqueología de Patagonia: una mirada desde el último confín, editado por M. Salemme, F. Santiago, M. Álvarez, E. Piana, M. Vázquez y M.E. Mansur. Editorial Utopías, Ushuaia. En prensa.

PIGEOT, N. 2003. L'Économie de la taille de la pierre. En: Cazadores-Recolectores de Ponsonby (Patagonia austral) y su paleoambiente desde VI al III milenio A.C. editado por D. Legoupil, pp. 117-164. Universidad de Magallanes MAGALLANIA Vol. 31, número especial, Punta Arenas.

SAN ROMÁN, M.; M. FONTUGNE Y K. SALAS. 2009. Primeros avances en la reconstrucción de secuencias de ocupación de cazadores recolectores marinos en el estrecho de Magallanes, Patagonia meridional. En: Arqueología de Patagonia: una mirada desde el último confín, editado por M. Salemme, F. Santiago, M. Álvarez, E. Piana, M. Vázquez y M.E. Mansur. Editorial Utopías, Ushuaia. En prensa. 
